\title{
Detecting Weak Targets in a Speckled Distributed Scene by SAR Reconfiguration
}

\author{
Kiyo Tomiyasu \\ Formerly Lockheed Martin Corporation \\ Retired, now Pomona, California, USA \\ ektom2@verizon.net
}

\author{
Rolf Scheiber \\ German Aerospace Center (DLR) \\ Microwaves and Radar Institute \\ 82230 Wessling, Germany \\ rolf.scheiber@dlr.de
}

\begin{abstract}
In this paper a discussion on the difference between point-like targets and distributed scene in SAR images is presented starting from basic radar equations. The target detection requirements are formulated considering also the speckle characteristics of the distributed scattering of the background. An equation is presented for the estimation of the minimum radar cross section of a target to become detectable within a scene with speckled distributed background and detection criteria are discussed for the multi-look case. Experimental evaluations are based on airborne SAR data of a calibration test site.
\end{abstract}

\section{INTRODUCTION}

Point targets such as a trihedral and small specular objects located in a low-backscatter scene, for example a dry lake, have been imaged clearly isolated, and such images are useful in calibrating synthetic aperture radars [1]. Trihedrals located in a forest have also been imaged but these trihedrals are not as clearly recognized because of strong backscattering from the surrounding forest [2]. Besides trihedrals, objects such as a vehicle may be difficult to discern if located in a distributed scene with relatively strong backscatter. In this paper, evaluations are reported that show enhancements in contrast by reconfiguring the SAR.

The accuracy of using point targets to calibrate synthetic aperture radar (SAR) has been discussed [3]. A recent paper discusses an adaptive method to extract urban area features from a SAR [4]. Another recent paper discusses improving SAR range resolution by combining two images from slightly different viewing angles [5]. A more complex problem is detecting camouflaged complex targets [6].

In this paper the SAR performances are briefly analyzed for the detection of point targets like trihedrals and the examination of the conditions that would improve recognition of point targets located in an area of strong distributed scattering. Expressions for the minimum radar cross section are derived for the single look and multi-look cases. The discussion is accompanied by experimental results performed by the German Aerospace Center airborne E-SAR sensor imaging a trihedral reflector located in a grass field in Oberpfaffenhofen, Germany.

\section{DiSTRIBUTED SCATTERERS}

Small point or specular targets located within a strong distributed scattering area of a SAR image with speckle can be difficult to discern and characterize [7]. The mean value of the backscattered return per pixel of a distributed scene $\sigma_{\text {Mdistr }}$ with dimensions of square meters was introduced and nominally defined as [10],[11]:

$$
\sigma_{\text {Mdistr }}=\sigma^{0} \delta_{r} \delta_{a z} / \sin \theta_{i}
$$

where $\delta_{a z}$ is azimuth resolution, $\delta_{r}$ is slant range resolution, $\theta_{i}$ is incidence angle, and thus $\delta_{r} \delta_{a z} / \sin \theta_{i}$ is the resolution area on ground. The quantity $\sigma^{o}$ is dimensionless, and is the radar backscattering coefficient of the scene (sometimes also termed normalized radar cross section). This coefficient is dependent on the sensor wavelength and polarization as well as scene geometry and terrain parameters. A good compilation of $\sigma^{\circ}$ of many scenes is given by Ulaby and Dobson [10]. The slant range resolution of the SAR is usually fixed and is related to pulse bandwidth. However to achieve the azimuth resolution, the radar platform must travel some distance while emitting pulses, that are coherently related, in order to create the "synthetic aperture" through computer processing.

Within each pixel area of $\delta_{r} \delta_{a z} / \sin \theta_{i}$ there are numerous scatterers. Each scatterer will have a backscatter amplitude, phase and polarization. The complex returns from all scatterers in a resolution cell are coherently added. This results in a speckle effect that is quantified by the ratio of standard deviation of the fluctuations to the mean value, $\sigma_{\text {Mdistr }}$, where the subscript $\mathrm{M}$ signifies the mean value of all pixels.

In order to image the distributed scene with sufficient signal-to-noise ratio, the radar must have sufficient average power, $P_{\text {ave }}[11],[12]$ : 


$$
S / N=\frac{P_{a v e} A_{r}^{2} \eta^{2} \delta_{r} \sigma^{0}}{8 \pi R^{3} k T_{r} N F v \lambda L_{S} \sin \theta_{i}}
$$

where $S$ is signal power, $N$ is noise power, $P_{\text {ave }}$ is average radar power, $A_{r}$ is radar antenna area, $\eta$ is antenna efficiency, $\delta_{r}$ is slant range resolution, $\sigma^{o}$ is backscattering coefficient of the distributed scene, $R$ is radar range, $k$ is Boltzmann's constant, $T_{r}$ is receiver absolute temperature, $N F$ is relative noise figure, $v$ is radar velocity, $\lambda$ is radar wavelength, and $L_{s}$ is total system loss.

\section{Point TARget Detection}

\section{A. General discussion}

A point target, such as a trihedral, has over a wide angular sector, a fixed radar cross section, $\sigma_{p t}$ with units of square meters that is independent of its orientation over a substantial range, and the azimuth and range resolutions of the radar. It is further assumed that there is one point target in one pixel defined by $\delta_{r}$ and $\delta_{a z}$. To be detected, the point target radar cross section is assumed dimensionally equivalent to and defined as:

$$
\sigma_{p t}=\sigma_{p t}^{0} \delta_{r} \delta_{a z}
$$

or rewritten, $\sigma_{p t} / \delta_{a z}=\sigma_{p t}^{0} \delta_{r, \text {, where }} \sigma_{p t}$ is the backscattering coefficient of the point target [10].

The fundamental signal-to-noise equation for imaging a point target by a SAR is derived from (2) and rewritten for the point target with cross section, $\sigma_{\mathrm{pt}}$ as:

$$
S / N=\frac{P_{a v e} A_{r}^{2} \eta^{2} \sigma_{p t}}{8 \pi R^{3} k T_{r} N F v \lambda L_{S} \delta_{a z}}
$$

Note the $\mathrm{S} / \mathrm{N}$ and hence the required SAR transmitter power for detecting point targets by SAR still depends on $\mathrm{R}^{3}$, and not $\mathrm{R}^{4}$ as for a pencil-beam radar. One use of SAR is to locate point targets in the presence of speckle from its surrounding distributed scattering area. Sufficient SAR power is needed to detect the point target and to assess the surrounding distributed scene. However there remain two other problems, one is the speckle of distributed scenes. The confusing speckle effect can be reduced by adding multiple looks taken of the scene. The other is the power required to determine the SAR response of the point, or specular target. The mean value of the distributed scattering is characterized by $\sigma^{0}$ times the ground range and azimuth resolutions.

To compare point target detection with distributed scatterer detection, the $\mathrm{S} / \mathrm{N}$ for a point target after removing common terms is given by:

$$
(S / N)_{p t} \propto P_{a v e} \sigma_{p t} / \delta_{a z}
$$

It is noted that the $\mathrm{S} / \mathrm{N}$ of a point target improves with a decrease in $\delta_{a z}$. This is due to a longer integration time. Next, for a nominal distributed scene, neglecting speckle,

$$
(S / N)_{\text {Mdistr }} \propto P_{\text {ave }} \delta_{r} \sigma^{0} / \sin \theta_{i}
$$

This equation shows that the return from a nominal distributed scene can become weaker with a finer range resolution. To detect a point target located in a distributed scene with a given radar power $P_{\text {ave }}$ the $S / N$ of these two scenarios are compared for contrast. The integrated noise power $N$ of the SAR is the same for both point target and diffuse scene. The transmit power, $P_{\text {ave }}$ is the same for both scenes. Hence the ratio of the $S_{p t}$ and median $S_{M d i s t r}$ signals, neglecting speckle, is:

$$
\frac{S_{p t}}{S_{\text {Mdistr }}}=\frac{\sigma_{p t} \sin \theta_{i}}{\sigma^{0} \delta_{r} \delta_{a z}}
$$

This can be referred to the signal-to-mean clutter ratio.

This formulation states that point targets located in a diffuse scene will be more pronounced if either or both azimuth and range resolutions are improved. To achieve the azimuth resolution, the beam direction $\theta$ is changed during integration, and the dwell angle, is given by:

$$
\Delta \theta=(\lambda / 2) / \delta_{a z}
$$

Alternatively, the azimuth resolution can be improved by using a shorter wavelength radar:

$$
\delta_{a z}=(\lambda / 2) / \Delta \theta
$$

If the SAR has a multi-look operation to reduce speckle, the entire data set can be processed for single-look and improve $\delta_{a z}$.

\section{B. Experimental demonstration}

The airborne E-SAR system of DLR (German Aerospace Center) was provided and flown in an aircraft, at an altitude of 3200 meters with a speed of 91.4 meters $/ \mathrm{sec}$. The equipment descriptions are:

TABLE I. E-SAR SYSTEM PARAMETERS AT L-BAND

\begin{tabular}{|c|l|c|l|}
\hline Center Freq. & $1300 \mathrm{MHz}$ & Range & $5000 \mathrm{~m}$ \\
\hline Peak Power & $56 \mathrm{Watt}$ & Azimuth resol. & $>0.4 \mathrm{~m}$ \\
\hline Bandwidth & $100 \mathrm{MHz}$ & Range resolution & $>1.5 \mathrm{~m}$ \\
\hline PRF & $400 \mathrm{~Hz}$ & Chirp Length & $5 \mu \mathrm{sec}$ \\
\hline Polarization & $\mathrm{VV}$ & Flight altitude & $3200 \mathrm{~m}$ \\
\hline Antenna Width & $0.7 \mathrm{~m}$ & Grazing Angle & $35 \mathrm{deg}$ \\
\hline Antenna Height & $0.35 \mathrm{~m}$ & Dwell angle & $18 \mathrm{deg}$ \\
\hline
\end{tabular}

a. E-SAR is a multi-frequency polarimetric airborne SAR. Here only L-band VV is used. 

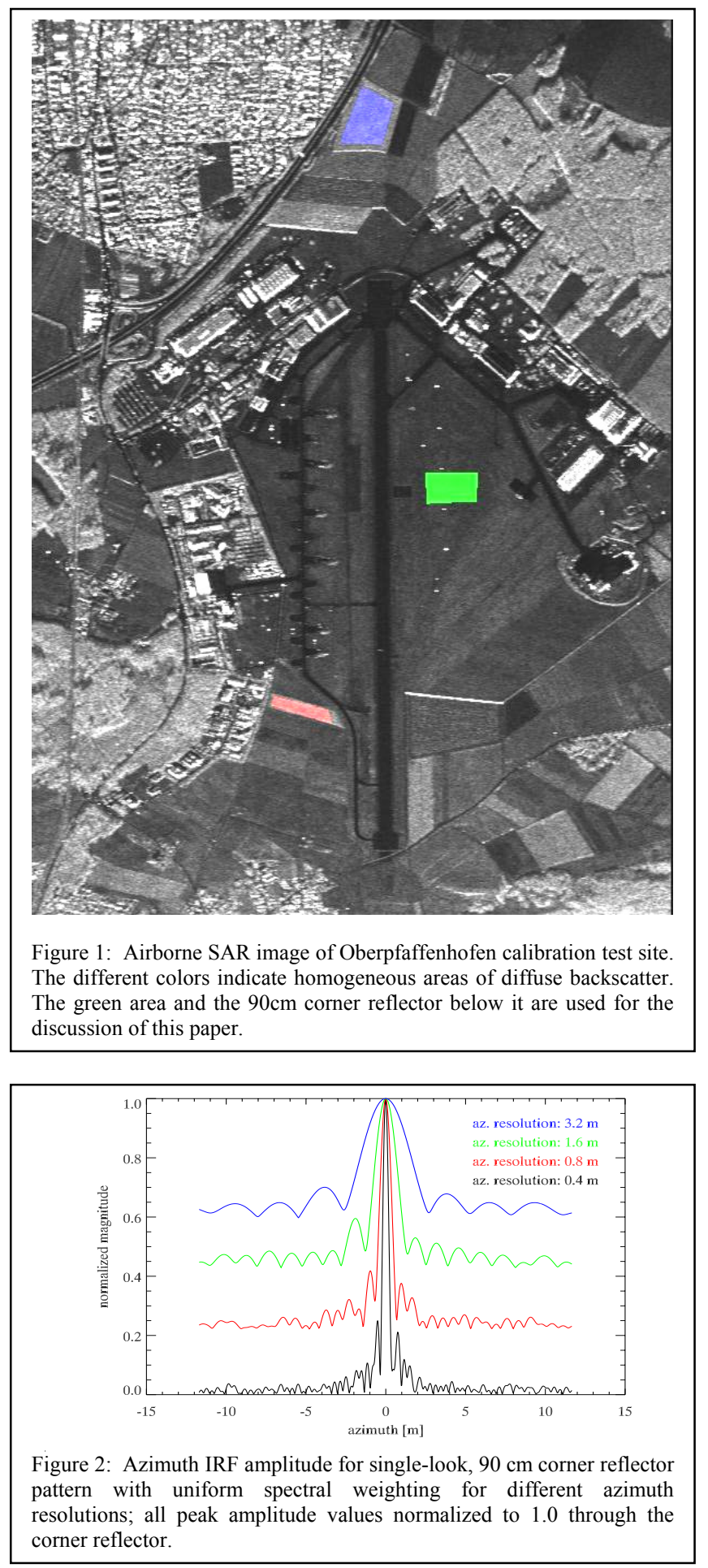

The SAR image of size $2 \mathrm{~km} \times 3 \mathrm{~km}$ is depicted in Figure 1 including trihedral reflectors as calibration targets. To demonstrate the benefit of improved azimuth resolution in terms of contrast enhancement between a $90-\mathrm{cm}$ trihedral reflector and its surrounding, Figure 2 is shown. It is easily observed, that for all resolutions the trihedral maximum is well above the homogeneous clutter, that corresponds to $\sigma^{0}=-15 \mathrm{~dB}$. This is desired, as for calibration targets the resolution and the sidelobe levels want to be evaluated. (Azimuth sidelobes can be observed in the vicinity of the main lobe.) The finest resolution curve also indicates at further distances from the peak, a statistical signal corresponding to the background. The magnitude of these "speckle" fluctuations from pixel to pixel may be in the same order of the mean value itself, of the distributed surface, but the speckle could be more noticeable and more objectionable compared to the mean. It is noted that the contrast between the trihedral and diffuse scene responses becomes greater as the azimuth resolution improves, and also speckle becomes less objectionable. Other than increasing the resolution, it is also possible to reduce speckle by multi-looking to enhance the point targets located within a distributed scene. A detailed discussion on the implication of the speckle effect is given in the next two sections.

\section{SPECKLE CONSIDERATIONS}

In a typical homogeneous area, the random spatial distribution of the scatterers results in intra-pixel interferometry and there is a significant change in total vector scattering from pixel to pixel in the apparent "uniform" scene. This is the origin of speckle.

Usually, the statistical complex backscatter from a distributed scene is modelled as a Rayleigh (circular Gaussian) process. Therefore its intensity $I$ follows the exponential distribution with equal mean and standard deviation [1],[10],[11]. The consequence is that a point target, if not sufficiently strong, might become obscured due to strong speckle from the surrounding background even if the backscattering coefficient $\sigma_{\text {pt }}^{0}$ (see eq. 3) is larger than the $\sigma^{0}$ of the surrounding background. (Note that a new variable $I$ is introduced to make a clear distinction between the statistical nature of the backscattering coefficient and its mean value $\sigma^{0}$.)

For illustration, the histogram of intensity values corresponding to the green area in the vicinity of the corner reflector is depicted in Figure 3. The density function follows closely the theoretically expected exponential decay and the mean value is indicated by the green line. It is observed, that due to speckle, a considerable amount of pixels come with intensities larger than the mean normalized radar cross section, which is annotated as the $\sigma^{0}$ of this area. The magenta diamond relates to the expected normalized radar cross section of the $90 \mathrm{~cm}$ trihedral with $\mathrm{RCS}=17 \mathrm{dBsqm}$, if imaged with a resolution of $15 \mathrm{~m}$ by $15 \mathrm{~m}$. If the resolution of the SAR system is coarser, or the RCS of the point target becomes smaller, the detection will be obscured by the speckle background, as the histogram and the strong target return will shift closer together.

In Figure 4 the same histogram is shown with logarithmic abscissa for increased dynamic range. Colored diamonds are used to represent the backscattering coefficient of the trihedral for the different resolutions shown in Figure 2. (i.e. the intensity of the corner reflector response). 


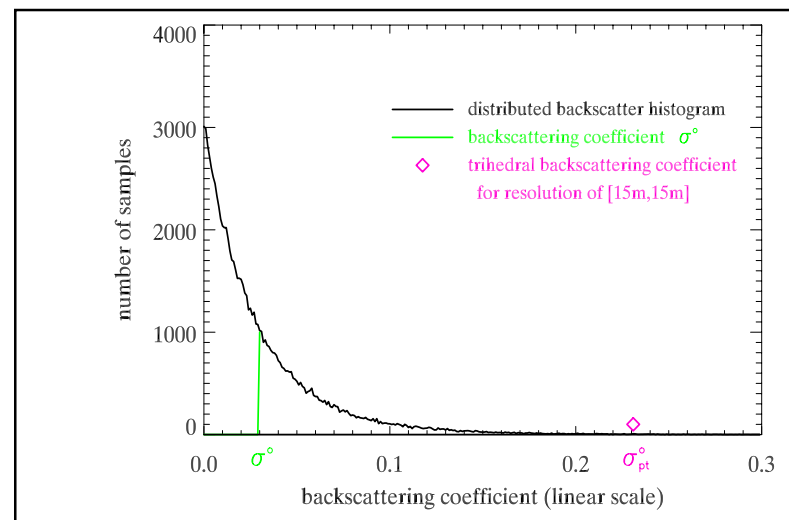

Figure 3: Histogram of grass area surrounding the trihedral reflector location. The backscattering coefficient of this area is indicated by the green line. For comparison the backscattering coefficient of the $90 \mathrm{~cm}$ trihedral is shown in magenta for a resolution of $15 \mathrm{~m} \times 15 \mathrm{~m}$.

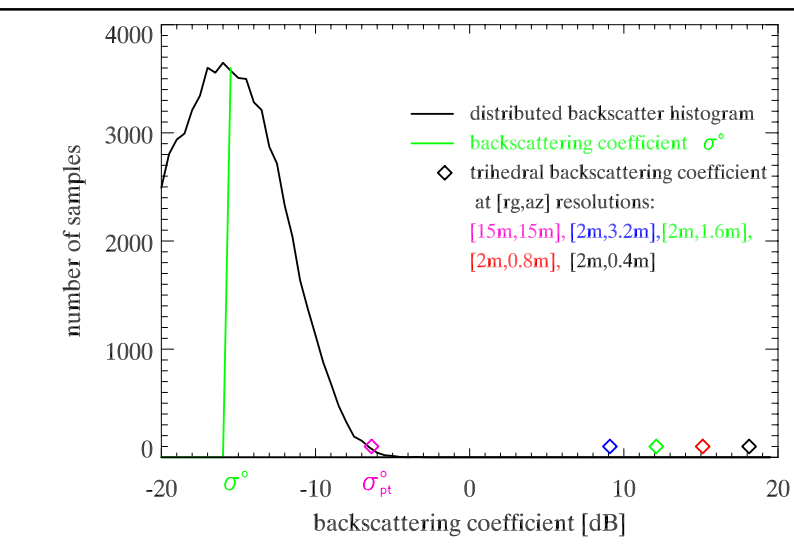

Figure 4: Histogram of grass area surrounding the trihedral reflector location (same area as for Figure 3). The increased dynamic range of the logarithmic scale allows the representation of the trihedral backscattering coefficient for different resolutions.

The response of a point target is assumed as a rectangle with the size of the resolution and with intensity of $\sigma_{\mathrm{pt}} /\left(\delta_{\mathrm{az}} \delta_{\mathrm{r}}\right)$. The best detection of the point target is achieved for the finest resolution. It is observed that the contrast enhances by $3 \mathrm{~dB}$ for every improvement in resolution of factor 2 .

Now, a general formulation is provided for the detection of a weak target in a speckled scene with mean backscattering coefficient $\sigma^{0}$. The effect caused by speckle in SAR performance is similar to "false" response in a conventional pencil beam radar due to returns from terrain [13]; this is treated by a CFAR (constant false alarm ratio) approach. For SAR, the detection failure probability $D_{f}$ is imposed:

$$
1-\int_{0}^{\sigma_{p t, \min }^{0}} \frac{1}{\sigma^{0}} e^{-\frac{I}{\sigma^{0}}} d I<D_{f}
$$

This equation integrates the probability density function (PDF) of the normalized backscatter intensity $I$ (histograms are shown in Fig.3 and 4). $\sigma_{\mathrm{pt} \text {,min }}^{0}$ is the quantity to be estimated and corresponds to the minimum backscattering coefficient of the point target to become detectable. Fortunately, eq. (10) can be easily solved for $\sigma_{\mathrm{pt} \text {,min }}^{0}$, when knowing the backscattering coefficient $\sigma^{0}$.

$$
\sigma_{p t, \min }^{0}>-\sigma^{0} \cdot \ln \left(D_{f}\right)
$$

Finally, $\sigma_{\mathrm{pt} \text { min }}^{0}$ is related to the minimum RCS of the pointlike target via:

$$
\sigma_{p t, \min }=\sigma_{p t, \min }^{0} \cdot \delta_{r} \delta_{a z} / \sin \theta_{i}
$$

Imposing a detection failure probability $D_{f}=10^{-4}$ means to allow one sample out of an area of 100 by 100 pixels to be larger than $\sigma_{\mathrm{pt} \text { min. }}^{0}$. Evaluating eq. (11) and (12) for the example area of section III $\left(\sigma^{0}=-15 \mathrm{~dB}\right)$ and the ground resolution of $15 \mathrm{sqm}$ the minimum radar cross section is found to be $18 \mathrm{dBsqm}$, which is slightly higher than the $17 \mathrm{dBsqm}$ of the investigated trihedral in Figures 3 and 4.

For a given $D_{f}$, the detection can be improved by improving the resolution of the sensor or by increasing the RCS of the point target. Particularly for radiometric radar calibration, the RCS of the calibration target should allow for $\sim 20 \mathrm{~dB}$ above the computed $\sigma_{\mathrm{pt} \text {,min }}^{0}$. For relating the RCS to different calibration targets and for calibration issues in general, see reference [1].

\section{Detection in the Multi-Look Case}

Several possibilities exist to reduce the influence of speckle on SAR images [9]. Here we evaluate how the multilook operation performed as a mean of intensities of multiple independent looks affects target detection, looks being assumed independent (e.g. generated in the spectral domain without overlap).

The PDF of an N-look image $p_{N}$ is described by a chisquare distribution with $2 \mathrm{~N}$ degrees of freedom [14]. For integer $\mathrm{N}$ the analytical expression is given as [9][10]:

$$
p_{N}\left(I ; \sigma^{0}\right)=\frac{I^{N-1} \cdot N^{N}}{(N-1) ! \sigma^{0}} e^{-N \frac{I}{\sigma^{0}}}
$$

The standard deviation of this density function decreases with $1 / \sqrt{N}$ and the mean is $\sigma^{0}$. For areas of homogeneous backscatter there is a good correspondence with the analyzed airborne data as shown in Figure 5.

The task of detecting a weak target within a distributed background of a multi-look image can now be analyzed as in the single-look case. This time the detection failure probability $D_{f}$ is imposed to the PDF of eq. (13). The minimum backscattering coefficient of the point target $\sigma_{\mathrm{pt}, \mathrm{min}, \mathrm{N}}^{0}$ to become detectable in a multi-look image of $N$ looks can thus be evaluated. 


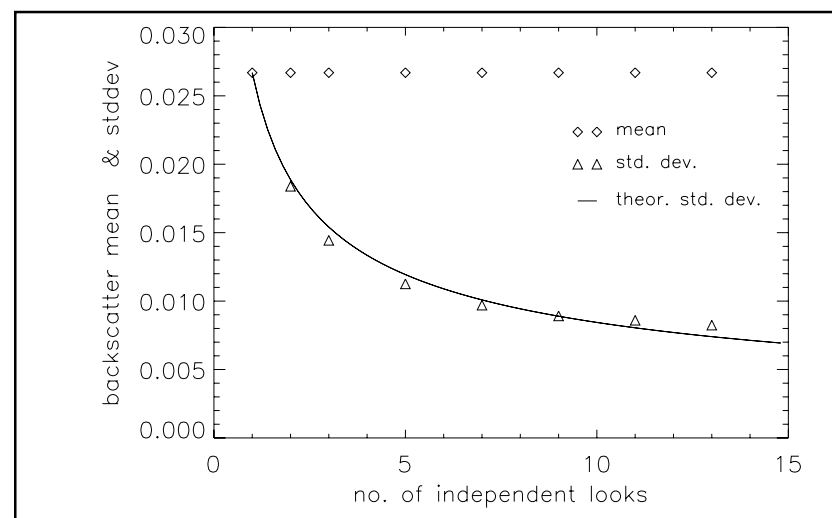

Figure 5: Backscatter intensity mean and standard deviation for different number of looks corresponding to green distributed area of airborne data depicted in Figure 1.

$$
1-\int_{0}^{\sigma_{p t, \min , N}^{0}} p_{N}\left(I ; \sigma^{0}\right) d I<D_{f}
$$

To solve for $\sigma_{\mathrm{pt}, \mathrm{min}, \mathrm{N}}^{0}$, first the distributions $F_{N}(I)$ of the PDFs in eq. (13) are evaluated for the given integer number of looks $N$. This can be performed analytically (which involves an iterative approaches with $N$ iterations) or by numerical integration. The analytical evaluation of the distribution function for different number of looks $N$ has been performed and is presented in Figure 6 assuming $\sigma^{0}=1$.

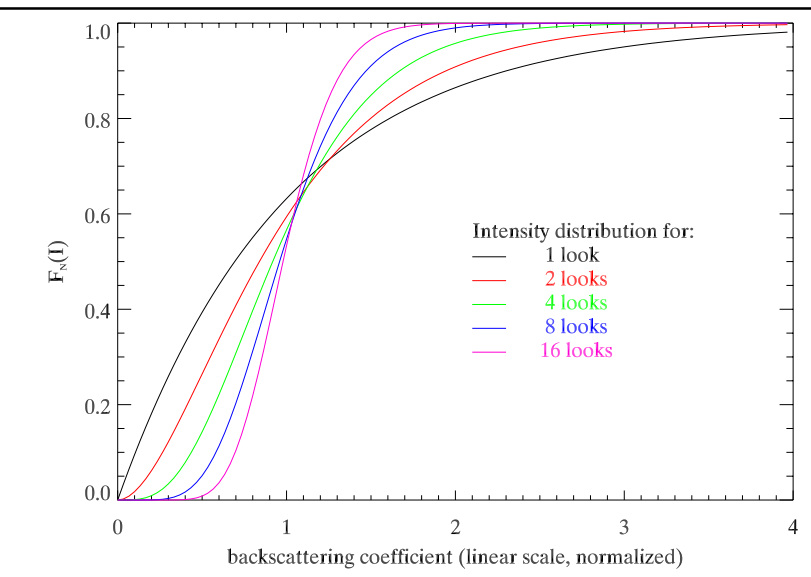

Figure 6: Distribution function of multi-look intensity for different number of looks $\mathrm{N}$. The backscattering coefficient of the distributed area is normalized to one.

Next, these distribution functions can directly be used to evaluate $\sigma_{\mathrm{pt,min,N}}^{0}$. They correspond to the integral in eq. (14). In Figure 7 is shown the minimum backscattering coefficient of a point target to be detected in a distributed scene of mean backscattering coefficient $\sigma^{0}=1$. A detection failure probability of $D_{f}=10^{-4}$ has been assumed. As the number of looks increases, the required $\sigma_{\mathrm{pt,min}, \mathrm{N}}^{0}$ of the target becomes less. A reasonably good approximation to this behavior is given by the square root decay with $\mathrm{N}$ (red line). We can therefore assume:

$$
\sigma_{p t, \min , N}^{0} \approx \sigma_{p t, \min }^{0} / \sqrt{N}
$$

Finally, the required radar cross section for the multi-look case can be computed as in the single look case (see eq. (12)).

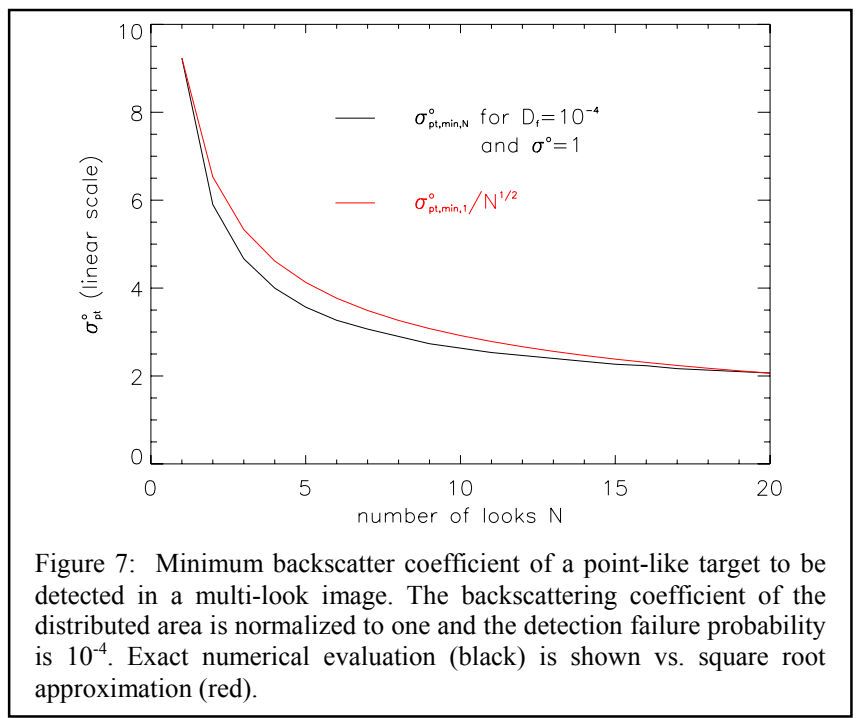

However, special care must be taken with respect to the resolution of the multi-look image, which needs to be considered instead. Usually multiple looks are obtained from one single-look image. In this case the resolution will be degraded (increased) by a factor of $\mathrm{N}$ :

$$
\begin{aligned}
& \sigma_{p t, \min , N}=\sigma_{p t, \min , N}^{0} \cdot \delta_{r} \delta_{a z} N / \sin \theta_{i} \\
& \approx \sigma_{p t, \min }^{0} \cdot \delta_{r} \delta_{a z} \sqrt{N} / \sin \theta_{i}
\end{aligned}
$$

For the same detection failure probability, the minimum RCS of a point target becomes larger the more number of looks are used. Best detection probability is found for the full resolution single-look case.

Alternatively, one could improve detection using multiple full resolution data sets acquired as temporal series or from considerably different aspect angles. Both cases ensure perfect point target coherence and at the same time decorrelation (statistical independence) of the distributed background. In this case the minimum required radar cross reduces to:

$$
\sigma_{p t, \min , N, \text { multi }} \approx \sigma_{p t, \min }^{0} / \sqrt{N} \cdot \delta_{r} \delta_{a z} / \sin \theta_{i}
$$

where the subscript multi accounts for multiple acquisitions. Stacks of data sets with aforementioned properties are often acquired by space-borne sensors for differential 
interferometric scatterers [15].

\section{CONCLUSIONS}

The SAR imaging of point-like targets and distributed areas was discussed in this paper. The detection requirements were obtained by contrasting the radar equation for these two cases. In a further step, the effects of speckle have been analyzed and a new equation has been formulated, which allows quantifying the minimum RCS for a target to be detectable in a speckled scene of given reflectivity. This formulation is extremely useful for the selection and tailoring of calibration targets for multi-frequency sensors with different resolution capabilities and for different clutter environments. It was further shown that multi-looking at the expense of spatial resolution does not improve the detection performance. However, detection can be improved if the multiple looks are obtained from multiple full resolution independent observations with uncorrelated backscattering from the distributed area surrounding the point target under consideration.

\section{ACKNOWLEDGMENT}

The authors wish to acknowledge the interest and guidance provided by Professor Alberto Moreira.

\section{REFERENCES}

[1] A. Freeman, "SAR calibration: a review," IEEE Trans. Geosci. Remote Sensing, vol. 30, pp. 1107-1121, Nov. 1992.

[2] K. Sarabandi, L.E. Pierce, M.C. Dobson, F.T. Ulaby, J.M. Stiles, T.C. Chiu, R. DeRoo, R. Hartikka, A. Zambetti and A. Freeman, "Polarimetric calibration of SIR-C using point and distributed targets," IEEE Trans. Geosci. Remote Sensing, vol. 33, pp. 858-866, July 1995.

[3] L.M.H. Ulander, "Accuracy of using point targets for SAR calibration," IEEE Trans. Aerospace and Electronic Systems, vol. 27, pp. 139-148, January 1991.

[4] C. He, G. Xia and H. Sun, "An adaptive and iterative method of urban area extraction from SAR images," IEEE Geosci. Remote Sens. Lett., vol. 3, pp. 504-507, Oct. 2006.

[5] S. Guillaso, A. Reigber, L. Ferro-Famil, and E. Pottier, "Range resolution improvement of airborne SAR images," IEEE Geosci. Remote Sens. Lett., vol. 3, pp. 133-139, Jan. 2006.

[6] M. Dehmollaian and K. Sarabandi, "Electromagnetic scattering from foliage camouflaged complex targets," IEEE Trans. Geosci. Remote Sensing, vol. 44, pp. 2698-2709, October 2006.

[7] L.J. Porcello, N. G. Massey, R. B. Innes, and J. M. Marks, "Speckle reduction in synthetic-aperture radars," J. Opt. Soc. Am., vol. 66, pp. 1305-1311, November 1976.

[8] A. Moreira, "Improved multilook techniques applied to SAR and SCANSAR imagery," IEEE Trans. Geosci. Remote Sensing, vol. 29, pp. 529-534, July 1991.

[9] J.S. Lee, I. Jurkevich, P. Dewaele, P. Wambacq and A. Oosterlinck, "Speckle filtering of synthetic aperture radar images: A review," Remote Sensing Reviews, Harwood Academic Publishers, 1994, vol. 8, pp. 313-340.

[10] F.T. Ulaby and M.C. Dobson, HANDBOOK OF RADAR SCATTERING STATISTICS FOR TERRAIN radar scattering statistics for terrain, Boston:Artech House, 1981, $357 \mathrm{p}$.

[11] J.C. Curlander and R.N. McDonough, SYNTHETIC APERTURE RADAR, systems and signal processing, New York:John Wiley \& Sons, $1991,647 \mathrm{p}$.
[12] K. Tomiyasu, "Tutorial review of synthetic aperture radar (SAR) with applications to imaging of the ocean surface," Proc. IEEE, vol. 66, pp. 563-583, May 1978.

[13] M. Skolnik, RADAR HANDBOOK, Second Edition, Mc-Graw Hill; New York, 1990.

[14] A. Papoulis, PROBABILITY, RANDOM VARIABLES, AND STOCHASTIC PROCESSES, Mc-Graw Hill, New York, 1965.

[15] A. Ferretti, G. Savio, R. Barzaghi, A. Borghi, S. Musazzi, F. Novali, C. Prati, and F. Rocca: "Submillimeter Accuracy of InSAR Time Series: Experimental Validation” IEEE Trans. Geosci. Remote Sensing, vol. 45, no. 5, pp. 1142-1153, May, 2007. 